\title{
Brain Imaging in Pediatric Pseudotumor Cerebri Syndrome
}

\author{
Emanuele David $^{1,2}$ Kshitij Mankad ${ }^{3}$ \\ ${ }^{1}$ Department of Radiology, Anatomopathology and Oncology, \\ Sapienza University of Rome, Rome, Italy \\ 2 Department of Radiology, University of Messina, Messina, Italy \\ 3 Department of Neuroradiology, Great Ormond Street Hospital for \\ Children NHS Foundation Trust, London, United Kingdom

\begin{abstract}
Address for correspondence Kshitij Mankad, MBBS, MRCP, FRCR, Department of Neuroradiology, Great Ormond Street Hospital for Children, London, United Kingdom (e-mail: kshitij.mankad@gosh.nhs.uk; drmankad@gmail.com).
\end{abstract}

J Pediatr Neurol 2015;13:49-53.

\begin{abstract}
Keywords

- magnetic resonance imaging

- idiopathic intracranial hypertension

- pseudotumor cerebri

- venography

- transverse sinus stenosis

Pseudotumor cerebri syndrome (PTCS) is a clinical entity of unclear etiology characterized by raised intracranial pressure but normal cerebrospinal fluid contents and no associated anomalies evident in the brain parenchyma. Idiopathic intracranial hypertension, which is the primary (idiopathic) form of PTCS, generally affects adult obese females of childbearing age. Pediatric PTCS has been reported in association with various potential comorbidities, including chronic medical illness, infections, endocrine disorders, and ingestion and/or withdrawal of some drugs. Clinical signs and symptoms of PTCS in children are heterogeneous, but they classically include headache, papilledema, and visual disturbances (e.g., diplopia and/or vision loss). An important criterion for diagnosing PTCS involves imaging. The role of computed tomography is useful to rule out some secondary causes of elevated increased intracranial pressure (e.g., hydrocephalus, hemorrhage, mass effect); magnetic resonance imaging and magnetic resonance venography are helpful in many diagnostic challenges and in the detection of subtle intracranial anomalies (e.g., isodense tumors, meningeal infiltrations, pathologies of posterior fossa, sinus venous thrombosis). In addition, magnetic resonance imaging is able to identify some secondary signs supportive of a diagnosis of PTCS, such as flattened posterior globe/sclera, empty sella, partially empty sella/ decreased pituitary height, patulous optic nerve sheath, intraocular protrusion of optic nerve head, and enhanced and increased tortuosity of the optic nerve, though these are not specific. We briefly discuss the application of imaging in the assessment of pediatric PTCS patients.
\end{abstract}

\section{Introduction}

Pseudotumor cerebri syndrome (PTCS) is an umbrella term used for the constellation of symptoms caused by increased intracranial pressure (ICP) without hydrocephalus, spaceoccupying lesion, or anomalies of brain parenchyma and with a normal cerebrospinal fluid (CSF) composition. ${ }^{1}$ PTCS has a reported incidence of 0.9 per 100,000 in the general population, but it rises to 19 per 100,000 in obese women of childbearing age. ${ }^{2}$ In adults, primary PTCS, also known as idiopathic intracranial hypertension, tends to mainly affect overweight females of childbearing age who present with headache and papilledema. ${ }^{3}$

The term "primary" is reserved for those cases in which known secondary causes have been excluded. The term "secondary" is used for those cases in which an underlying potential cause is identified; current data suggest that prepubertal children affected with intracranial hypertension are more likely to be secondary rather than primary. ${ }^{4}$ In fact, received

October 30, 2014 accepted after revision January 21, 2015
Issue Theme Pediatric Pseudotumor Cerebri Syndrome; Guest Editors: Vincenzo Salpietro, MD, Martino Ruggieri, PhD, and Conrad E. Johanson, PhD
Copyright $\odot 2015$ by Georg Thieme Verlag KG, Stuttgart · New York
DOI http://dx.doi.org/ 10.1055/s-0035-1555155. ISSN $1304-2580$. 
systemic conditions, cerebral venous abnormalities, drugs, infections, and endocrine abnormalities, have been identified as possible secondary causes of PTCS in the pediatric literature, ${ }^{5-8}$ although the exact mechanism by which these conditions result in secondary intracranial hypertension is not entirely understood.

Clinically, the physical and neurologic examination should be normal with the exception of cranial nerve (e.g., abducens nerve) palsy (more frequent in children rather than in adults and typically a false localizing sign) and papilledema on funduscopic examination, which is present in almost all patients. The absence of papilledema or the presence of focal neurological deficits should put the diagnosis of PTCS in doubt. As weight gain and obesity are an important risk factor of PTCS in the pediatric age group, ${ }^{9}$ weight loss for overweight children may lead to a relief of clinical symptoms. ${ }^{10}$

Medical treatment (e.g., carbonic anhydrase inhibitors) is performed to reduce ICP and the severity of headache and visual disturbances in children with PTCS ${ }^{4,8,10}$; CSF diversion procedures (e.g., lumbar puncture [LP]) or surgical procedures (e.g., lumboperitoneal shunt, optic nerve sheath fenestration) are reserved for those patients who do not respond to conservative treatments or who had complications following diuretic administration. ${ }^{11}$ Although PTCS has been for long considered a benign condition, a small number of patients, especially if not promptly diagnosed and treated, can follow a more aggressive course, with permanent visual impairment occurring in one or both eyes in up to 8 to $10 \%$ of patients. ${ }^{12}$

Of note, the advent of magnetic resonance imaging (MRI) revolutionized the imaging paradigm in PTCS, from simply applying imaging to exclude concomitant intracranial processes (e.g., intracranial mass) to depicting signs thought to indicate and/or diagnose this syndrome itself (-Table 1). ${ }^{13}$ We briefly discuss on the increasingly emergent role of MRI in the assessment of pediatric PTCS patients.

\section{Initial Radiologic Assessment in a Child with Increased Intracranial Pressure}

The diagnosis of PTCS in children should be regarded always as a diagnosis of exclusion, as central nervous system tumors

Table 1 Key radiologic features of pseudotumor cerebri syndrome

\begin{tabular}{|l|}
\hline Radiological features \\
\hline Empty sella \\
\hline Partially empty sella \\
\hline Intraocular protrusion of optic nerve head \\
\hline Decreased pituitary height \\
\hline Flattened posterior sclera \\
\hline Enlarged perioptic subarachnoid space \\
\hline Increased tortuosity of optic nerve \\
\hline Transverse venous sinus stenosis \\
\hline Enhancement of optic nerve \\
\hline
\end{tabular}

and/or space-occupying lesions have to be first ruled out in all children who present with a constellation of symptoms, including headache, nausea, vomiting, and papilledema ${ }^{4}$; in this context, both computed tomography (CT) and MRI of the brain are extremely useful in the initial assessment of pediatric patients with increased ICP. ${ }^{4,8}$ As younger children with PTCS may develop torticollis, gait imbalance, and/or other clinical signs of posterior fossa lesion, MRI is useful also to exclude posterior fossa pathologies or postinflammatory torticollis. $^{14}$

A normal CT scan of the brain is never adequate in diagnosing PTCS or excluding its differentials. MRI of the brain (with and without gadolinium) and MR venography (MRV) are therefore the studies of choice to rule out known secondary causes. ${ }^{1,4,13}$ For instance, some infiltrating neoplastic processes (e.g., gliomatosis cerebri) may mimic PTCS, making crucial and careful MRI assessment in all cases of suspected PTCS. Leptomeningeal spread of lymphoma, leukemia, and germ cell tumors may also lead to signs and symptoms of intracranial hypertension in children, with only subtle anomalies on brain MRI. ${ }^{15,16}$ The diagnosis may be evident by meningeal enhancement on MRI. ${ }^{1,4}$ Furthermore, MRV is performed to exclude cerebral venous sinus thrombosis which can also mimic PTCS in clinical presentation. According to the recent diagnostic criteria, MRV should be performed in the initial radiologic assessment to exclude the presence of cerebral venous sinus thrombosis in those patients who do not fit the "typical" picture of primary PTCS (e.g., prepubertal children, not overweight patients, men). ${ }^{1}$

To diagnose PTCS, neuroimaging should demonstrate normal brain parenchyma without evidence of hydrocephalus, mass, or structural lesion and no abnormal meningeal enhancement. ${ }^{1,4,8}$ However, several abnormal MRI findings have been reported (i.e., empty sella, flattening of the posterior aspect of the globe, distention of the perioptic subarachnoid space with or without a tortuous optic nerve, transverse venous sinus stenosis). ${ }^{13,17}$ In this regard, brain imaging has acquired an important role in support to the diagnosis of PTCS, especially in those patients who have an incomplete presentation of the syndrome (i.e., without papilledema) but suggestive neuroimaging findings on MRI. ${ }^{1,4,8,13,17}$

\section{Imaging Findings in Pseudotumor Cerebri Syndrome}

According to the recent diagnostic criteria of PTCS in adults and children, ${ }^{1}$ a definitive diagnosis requires (1) papilledema, (2) a normal (excepting for cranial nerve abnormalities) neurologic examination, (3) a normal CSF composition, (4) an elevated LP opening pressure $\left(\geq 28 \mathrm{~cm} \mathrm{H}_{2} \mathrm{O}\right.$ in children or $\geq 25 \mathrm{~cm} \mathrm{H}_{2} \mathrm{O}$ in adults or in unsedated/nonobese children); and (5) the exclusion of secondary causes of raised ICP by brain imaging. According to diagnostic criteria, ${ }^{1}$ in the absence of papilledema or cranial nerve abnormalities (most commonly sixth nerve palsy), a diagnosis of PTCS can be suggested but not made if criteria 2 through 4 are satisfied and, additionally, if the patient meets at least three of the following (typical) PTCS-related imaging anomalies: (1) 


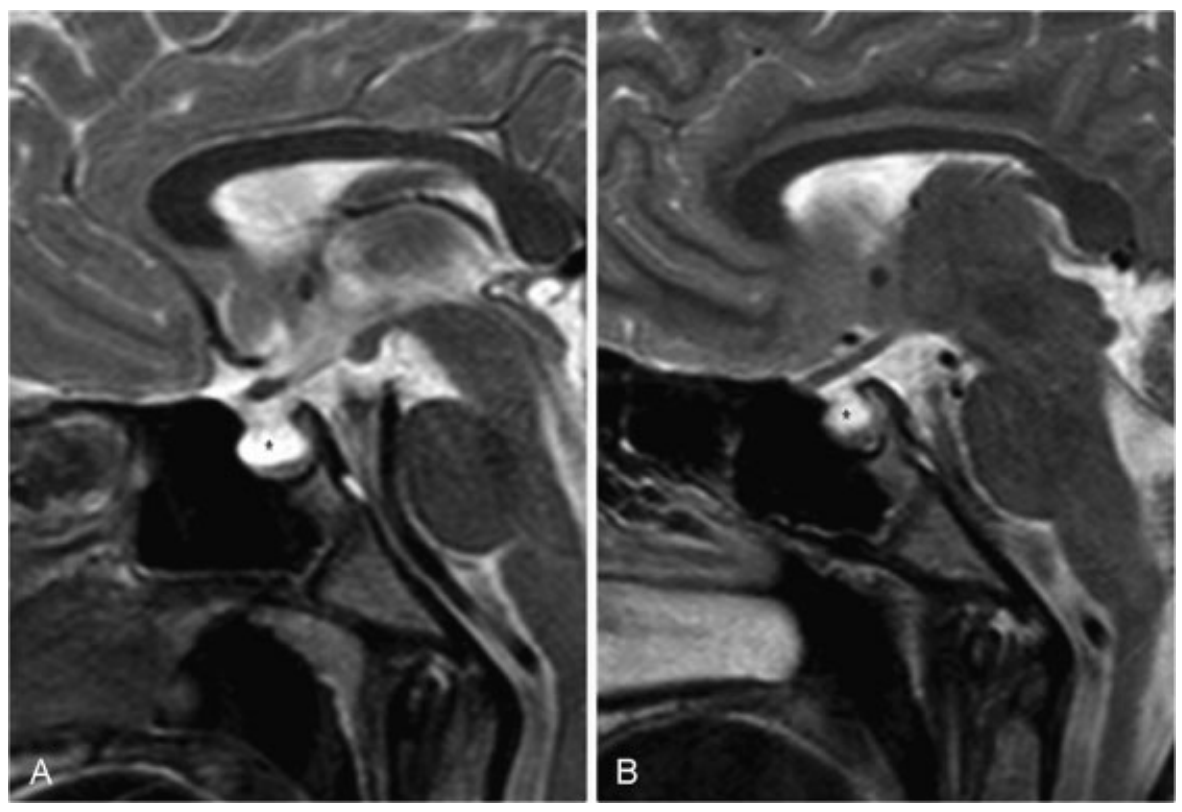

Fig. 1 (A, B) Sagittal fast-spin echo T2-weighted images show (asterisks) a partial empty sella.

empty sella, (2) flattening of the posterior aspect of the globe, (3) distension of the perioptic subarachnoid spaces (either associated or without a tortuous optic nerve), (4) transverse venous sinus stenosis. In fact, previous retrospective and/or prospective studies showed a significant incidence of specific radiologic findings in the context of PTCS. ${ }^{13}$

The presence of empty sella in PTCS patients represents the consequence of long-standing effects of increased ICP. Importantly, the term empty sella should be used to refer to the most severe cases, in which no pituitary gland can be identified on brain imaging. The incidence of concomitant increased ICP and empty sella is usually related to the severity and duration of PTCS and increase in the chronic PTCS group. ${ }^{18}$ While empty sella is a later manifestation of (severely) increased ICP, a moderate concavity of the pituitary gland without empty sella would suggest shorter duration and/or milder increase of CSF pressure. ${ }^{18}$ A preexisting diaphragmatic defect has been proposed as an important factor in making some patients with PTCS prone to develop empty sella. ${ }^{19}$ Increased ICP transmitted to the intrasellar subarachnoid space through an anatomic variant and/or anomaly (e.g., congenital or acquired defect of the diaphragm) likely results in remodeling of the sella turcica (and the nearly bone structures) and in compression of the pituitary gland. ${ }^{18,19}$

However, in the PTCS-related radiologic spectrum, there is a broad range of variations in pituitary height, and many cases of empty sella should be indeed regarded as partially empty sella or as compressed pituitary gland (-Figs. 1 and $\mathbf{2}$ ). The wide range of sensitivities and specificities reported reflects this ambiguity. ${ }^{13}$ Transverse sinus narrowing is frequently seen in MRV in patients with PTCS. A study ${ }^{20}$ identified stenotic transverse sinuses in $90 \%$ of patients with PTCS and the authors suggested, basing on the similar clinical
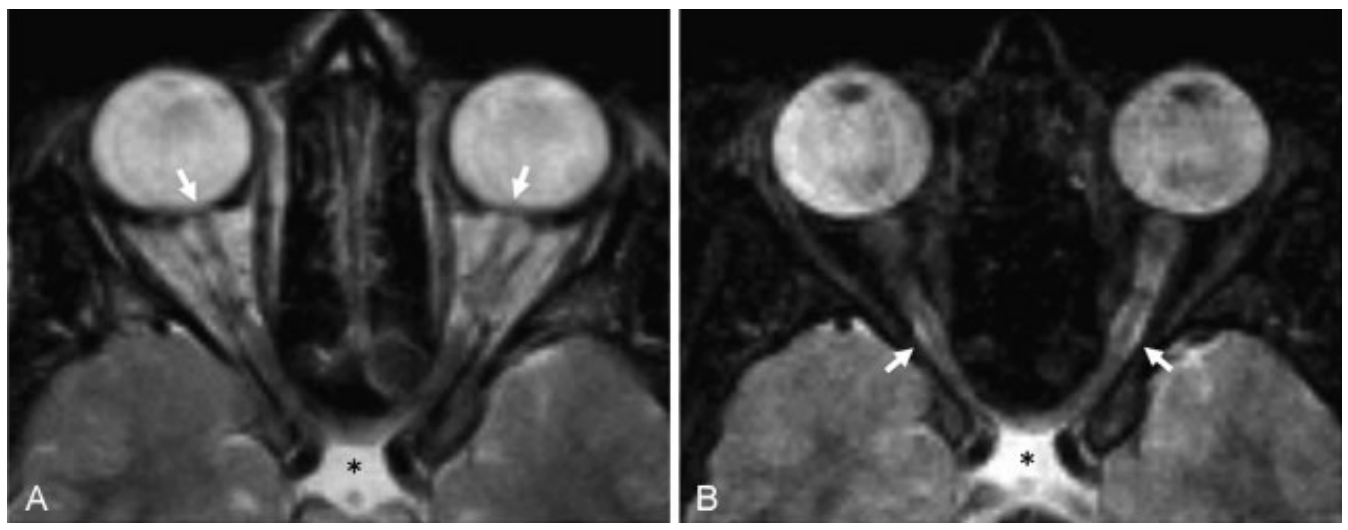

Fig. 2 Magnetic resonance imaging examination. (A, B) Axial T2-weighted fast-spin echo images of intraocular protrusion of optic nerve head (white arrows); enlarged perioptic subarachnoid space with cerebrospinal fluid hyperintensity surrounding the optic nerves (white arrows). The optic nerve sheath widening is thought to coincide with papilledema. Partial empty sella has been shown (black asterisk) in both images. 
presentation of "primary" PTCS and transverse sinus thrombosis, that most cases of PTCS are due to venous outflow obstruction, which can take the form of either thrombosis or stenosis. Even if it is assumed that venous stenosis exists in PTCS, there is still controversy as to whether venous stenosis and elevated venous pressure are due to elevated ICP or constitute the proximal cause of PTCS. ${ }^{21}$

In this respect, several authors suggested that an unsuspected previous abnormality, such as an anatomic variant (congenital narrowing) of the transverse sinus, can be present in a large number of asymptomatic healthy patients, ${ }^{21}$ and evidences from cadaveric studies also suggested that the stenosis of the transverse sinus observed in PTCS might be due to the presence of a large septum within the venous sinus. ${ }^{22}$ Thus, increased CSF pressure related to PTCS could exacerbate the underlying venous sinus abnormality and create a flow-limiting stenosis and resultant pressure gradient; this could worsen the elevation of the ICP. ${ }^{23}$ However, the compressible nature of the transverse sinus makes this structure vulnerable to tapering with increased ICP and this is now accepted by most authors as the effect (rather than the cause) of increased ICP. ${ }^{24}$ Posterior globe flattening could be regarded as one of the most frequent radiologic feature of PTCS and can be visualized on both CT and MRI. This sign presumably reflects the transmission of elevated perioptic CSF pressure to the compressible posterior sclera. ${ }^{25}$

Intraocular protrusion (-Fig. 2) likely occurring in a similar fashion to posterior globe flattening (i.e., due to elevated perioptic CSF pressure) and could be regarded as an infrequent radiologic feature associated with pediatric $\mathrm{PTCS}^{26}$; this finding on MRI imaging may well correspond to the presence of visual disturbances, because of its absence in patients with PTCS without visual symptoms. ${ }^{27}$ Widening of the optic nerve ( - Fig. 3 ) and optic nerve tortuosity have also been reported in pediatric PTCS, and both signs are considered to be related to increase ICP. ${ }^{25,26}$

\section{Perspectives on Self-Expanding Vascular Stents in Sinus Venous Stenosis}

An important developing therapeutic intervention for PTCS involves the use of self-expanding vascular stents in cases where there is evidence of sinus venous stenosis. This was first reported by Higgins et $\mathrm{al}^{28}$ with promising results, and other groups have also published results in which stenting of the transverse sinus was undertaken in the setting of idiopathic stenosis of the sinus. ${ }^{29}$ Overall, most patients showed improvement or complete resolution of the symptoms. In these studies, stenting significantly improved both the prestenotic transverse sinus pressures and pressure gradients. Furthermore, the results also showed improvement or resolution of papilledema in the majority of patients.

The results highlight that stenting in cases of isolated dural sinus stenosis may represent a successful alternative option to CSF diversion procedures. However, as outlined previously, the mechanism by which the stenosis occurs is unclear. It has been proposed that increased ICP itself was the major reason of venous sinus stenosis and it has been shown that regression of the stenosis after serial LPs, performed to decrease the ICP, can occur. ${ }^{23}$ Additionally, in an emblematic case study on PTCS associated with narrowing of the transverse sinuses, the removal of $20 \mathrm{~mL}$ of CSF led to a dimensional increase in the transverse sinuses with clinical remission after 2 months of follow-up. ${ }^{30}$

In conclusion, neuroimaging alone is not sufficient to diagnose PTCS, although some radiologic signs are highly suggestive of the syndrome. CT can identify secondary causes of intracranial hypertension, but MRI is the diagnostic gold standard in all patients suspected to have PTCS. In the MRI approach, it is important to first rule out subtle intracranial anomalies (e.g., infiltrating neoplastic processes). MRV allows detection of an underlying cerebral venous sinus thrombosis and has to be performed always in the atypical patients (e.g., not obese, not females). Sinus venous stenosis has been
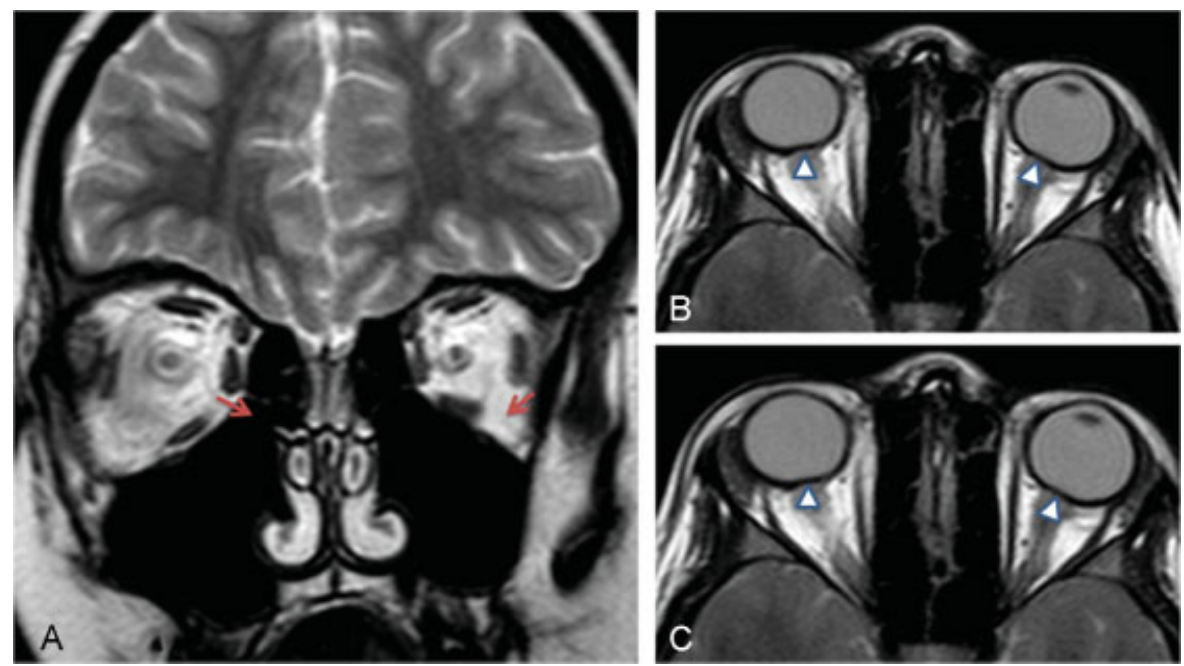

Fig. 3 (A) Coronal fast-spin echo T2-weighted image; (B, C) axial fast-spin echo T2-weighted images. Bilateral widening of the optic nerve sheath (red arrows). Bilateral optic papilla protrusion (arrowheads) with posterior globe flattening. 
frequently identified in PTCS, although this finding should be regarded as a consequence (rather than a cause) of increased ICP. The use of self-expanding vascular stents has been adopted in some PTCS patients with sinus venous stenosis on brain imaging and some authors propose that it may represent a successful alternative option to CSF diversion procedures. The evidence for this is, however, not absolute.

\section{Acknowledgments}

We gratefully acknowledge Dr. Francesca Granata who helped us in the preparation of the images contained in the present work.

\section{References}

1 Friedman DI, Liu GT, Digre KB. Revised diagnostic criteria for the pseudotumor cerebri syndrome in adults and children. Neurology 2013;81(13):1159-1165

2 Radhakrishnan K, Ahlskog JE, Cross SA, Kurland LT, O'Fallon WM. Idiopathic intracranial hypertension (pseudotumor cerebri). Descriptive epidemiology in Rochester, Minn, 1976 to 1990. Arch Neurol 1993;50(1):78-80

3 Khan MU, Khalid H, Salpietro V, Weber KT. Idiopathic intracranial hypertension associated with either primary or secondary aldosteronism. Am J Med Sci 2013;346(3):194-198

4 Rangwala LM, Liu GT. Pediatric idiopathic intracranial hypertension. Surv Ophthalmol 2007;52(6):597-617

5 Salpietro V, Polizzi A, Di Rosa G, et al. Adrenal disorders and the paediatric brain: pathophysiological considerations and clinical implications. Int J Endocrinol 2014;2014:282489

6 Salpietro V, Ruggieri M. Pseudotumor cerebri pathophysiology: the likely role of aldosterone. Headache 2014;54(7):1229

7 Salpietro V, Polizzi A, Bertè LF, et al. Idiopathic intracranial hypertension: a unifying neuroendocrine hypothesis through the adrenal-brain axis. Neuroendocrinol Lett 2012;33(6):569-573

8 Standridge SM. Idiopathic intracranial hypertension in children: a review and algorithm. Pediatr Neurol 2010;43(6):377-390

9 Salpietro V, Chimenz R, Arrigo T, Ruggieri M. Pediatric idiopathic intracranial hypertension and extreme childhood obesity: a role for weight gain. J Pediatr 2013;162(5):1084

10 Salpietro V, Mankad K, Kinali M, et al. Pediatric idiopathic intracranial hypertension and the underlying endocrine-metabolic dysfunction: a pilot study. J Pediatr Endocrinol Metab 2014; 27(1-2):107-115

11 Salpietro V, Ruggieri M, Sancetta F, et al. New insights on the relationship between pseudotumor cerebri and secondary hyperaldosteronism in children. J Hypertens 2012;30(3):629-630

12 Soiberman U, Stolovitch C, Balcer LJ, Regenbogen M, Constantini S, Kesler A. Idiopathic intracranial hypertension in children: visual outcome and risk of recurrence. Childs Nerv Syst 2011;27(11): 1913-1918
13 Degnan AJ, Levy LM. Pseudotumor cerebri: brief review of clinical syndrome and imaging findings. AJNR Am J Neuroradiol 2011; 32(11):1986-1993

14 Salpietro V, Polizzi A, Granata F, Briuglia S, Mankad K, Ruggieri M. Upper respiratory tract infection and torticollis in children: differential diagnosis of Grisel's syndrome. Clin Neuroradiol 2012; 22(4):351-353

15 Ebinger F, Brühl K, Gutjahr P. Early diffuse leptomeningeal primitive neuroectodermal tumors can escape detection by magnetic resonance imaging. Childs Nerv Syst 2000;16(7): 398-401

16 Zunz E, Ben Sira L, Constantini S, et al. Gliomatosis cerebri presenting as idiopathic intracranial hypertension in a child. J Neuroophthalmol 2011;31(4):339-341

17 Görkem SB, Doğanay S, Canpolat M, et al. MR imaging findings in children with pseudotumor cerebri and comparison with healthy controls. Childs Nerv Syst 2015;31(3):373-380

18 Yuh WT, Zhu M, Taoka T, et al. MR imaging of pituitary morphology in idiopathic intracranial hypertension. J Magn Reson Imaging 2000;12(6):808-813

19 Neelon FA, Goree JA, Lebovitz HE. The primary empty sella: clinical and radiographic characteristics and endocrine function. Medicine (Baltimore) 1973;52(1):73-92

20 Farb RI, Vanek I, Scott JN, et al. Idiopathic intracranial hypertension: the prevalence and morphology of sinovenous stenosis. Neurology 2003;60(9):1418-1424

21 Brazis PW. Pseudotumor cerebri. Curr Neurol Neurosci Rep 2004; 4(2):111-116

22 Subramaniam RM, Tress BM, King JO, Eizenberg N, Mitchell PJ. Transverse sinus septum: a new aetiology of idiopathic intracranial hypertension? Australas Radiol 2004;48(2):114-116

23 King JO, Mitchell PJ, Thomson KR, Tress BM. Manometry combined with cervical puncture in idiopathic intracranial hypertension. Neurology 2002;58(1):26-30

24 Corbett JJ, Digre K. Idiopathic intracranial hypertension: an answer to, "the chicken or the egg?" Neurology 2002;58(1):5-6

25 Brodsky MC, Vaphiades M. Magnetic resonance imaging in pseudotumor cerebri. Ophthalmology 1998;105(9):1686-1693

26 Mandelstam S, Moon A. MRI of optic disc edema in childhood idiopathic intracranial hypertension. Pediatr Radiol 2004;34(4): 362

27 Gass A, Barker GJ, Riordan-Eva P, et al. MRI of the optic nerve in benign intracranial hypertension. Neuroradiology 1996;38(8): 769-773

28 Higgins JN, Cousins C, Owler BK, Sarkies N, Pickard JD. Idiopathic intracranial hypertension: 12 cases treated by venous sinus stenting. J Neurol Neurosurg Psychiatry 2003;74(12):1662-1666

29 Bussière M, Falero R, Nicolle D, Proulx A, Patel V, Pelz D. Unilateral transverse sinus stenting of patients with idiopathic intracranial hypertension. AJNR Am J Neuroradiol 2010;31(4):645-650

30 De Simone R, Marano E, Fiorillo C, et al. Sudden re-opening of collapsed transverse sinuses and longstanding clinical remission after a single lumbar puncture in a case of idiopathic intracranial hypertension. Pathogenetic implications. Neurol Sci 2005;25(6): 342-344 